\title{
THE UNIQUE DECOMPOSITION OF REGULAR $\omega$-LINEAR MAPPINGS AS DIRECT PRODUCTS
}

\author{
N. J. S. HUGHES
}

We have previously [1] defined regular $n$-linear mappings and shown that they may be expressed in at most one way as direct products of indecomposable mappings. We wish to extend this result to the case in which $n$ is $\omega$ or, more generally, any transfinite ordinal $\alpha$ of class 2 .

If $H^{i}(i<\alpha)$ and $K$, which we sometimes denote by $H^{\alpha}$, are groups, $f$ is a regular $\alpha$-linear mapping on $H^{i}(i<\alpha)$ to $K$, denoted by

$$
f \in M\left(H^{i}, i<\alpha ; K\right) \text {, }
$$

if to every "vector" $\mathbf{x}=\left(x^{i}, i<\alpha\right), x^{i} \in H^{i}$, there is defined $f(\mathbf{x}) \in K$, and the following conditions are satisfied.

For any $i$, if $\mathbf{x}, \boldsymbol{y}$, and $z$ are such that

$$
\begin{gathered}
x^{j}=y^{j}=z^{j} \quad(\text { all } j \neq i), \\
x^{i} y^{i}=z^{i},
\end{gathered}
$$

then

$$
f(\mathbf{x}) f(\boldsymbol{y})=f(\boldsymbol{z}) .
$$

(2) If $x^{i} \in H^{i}$ is such that $f(y)=e$ (the identity), for all $y$ such that $y^{i}=x^{i}$, then $x^{i}=e$.

(3) The $f(x)$ generate $K$.

When $\alpha$ is transfinite, we use limiting processes.

For any $i \leqq \alpha$, for certain sequences $\left(x_{n}^{i}\right), x_{n}^{i} \in H^{i}, n=1,2,3, \cdots$, there is defined

$$
\lim _{n}\left(x_{n}^{i}\right) \in H^{i}
$$

If, for any $N, x_{n}^{4}=a$, for all $n>N$, then

$$
\lim _{n}\left(x_{n}^{i}\right)=a .
$$

We require $f$ to have the further property that, if, for all $i<\alpha$, $\lim _{n}\left(x_{n}^{i}\right)=x^{i}$, denoted by $\lim \left(x_{n}\right)=x$, then

$$
\lim _{n}\left(f\left(x_{n}\right)\right)=f(x) \text {. }
$$

Received by the editors May 30, 1951 . 
We know that, if $\alpha \geqq 2$, then $H^{i}$ is Abelian for all $i<\alpha$.

We say that $f$ is decomposable if, for all $i \leqq \alpha$,

$$
\begin{array}{cr}
H^{i}=\prod_{t \in T} H_{t}^{i} & \text { (direct product), } \\
x^{i}=\prod_{t \in T} x_{t}^{i}, \quad x_{t}^{i} \in H_{t}^{i}, & \text { uniquely, }
\end{array}
$$

and, if $i \neq j, t \in T, s \in T, t \neq s, x^{i} \in H_{l}^{d}, x^{j} \in H_{s}^{\prime}$, then

$$
f(\mathbf{x})=e,
$$

while, if, for all $i<\alpha, x^{i} \in H_{t}^{4}$, then

$$
f(\mathbf{x}) \in K_{t} .
$$

We denote $x_{t}^{t}$ in (7) by $x^{i} \theta_{t}$, so that $\theta_{t}$ is a projection operator, and we have

$$
\mathbf{x} \theta_{t}=\left(x^{i} \theta_{t}, i<\alpha\right) .
$$

Lemma 1. If $x^{0} \in H_{t}^{0}$, then $f(x)=f\left(x \theta_{t}\right)$.

For $n=1,2,3, \cdots$, we define $x_{n}$ by $x_{n}^{i}=x^{i}$, for $n \leqq i$, and $x_{n}^{i}=x^{i} \theta_{t}$, for $n>i$.

By induction, using (1), (7), and (8), we have, for all $n$,

$$
f\left(\mathbf{x}_{n}\right)=f(\mathbf{x}) .
$$

By (4) and (5),

$$
f\left(\mathbf{x} \theta_{t}\right)=f\left(\lim _{n}\left(\mathbf{x}_{n}\right)\right)=\lim _{n}\left(f\left(\mathbf{x}_{n}\right)\right)=f(\mathbf{x}) .
$$

This proves Lemma 1 when $\alpha=\omega$; if $\alpha>\omega$, the proof is completed by transfinite induction.

Lemma 2. $f(x)=\prod_{t \in T} f\left(x \theta_{t}\right)$.

This follows at once from (1), (7), and Lemma 1.

If $f_{t}$ is the mapping $f$ with the restricted range $x^{i} \in H_{i}$, then $f_{t}$ $\in M\left(H_{t}^{\prime}, i<\alpha ; K_{t}\right)$ and we express this by

$$
f=\prod_{t \in T} f_{t} .
$$

We now state our main result, proved previously [1] for finite $\alpha$.

Theorem 1. If $f=\prod_{t \in T} f_{t}, f_{t} \in M\left(H_{t}^{d}, i<\alpha ; K_{t}\right)$ and $f=\prod_{u \in U} f_{u}^{\prime}$, $f_{u}^{\prime} \in M\left(H_{u}^{\prime \prime}, i<\alpha ; K_{u}^{\prime}\right)$, then $f=\prod_{t \in T, u \in U} f_{t u}^{\prime \prime}, f_{t u}^{\prime \prime} \in M\left(H_{\imath}^{\prime} \cap H_{u}^{\prime i}, i<\alpha\right.$; $\left.K_{t} \cap K_{u}^{\prime}\right)$. 
Corresponding to (7), we have

$$
x^{i}=\prod_{u \in U} x^{i} \eta_{u}, \quad{H_{u}^{\prime}}^{i}=H^{i} \eta_{u}
$$

Then $x^{i}=\prod_{t \in T} x^{i} \theta_{t}=\prod_{t \in T, u \in U} x^{i} \theta_{t} \eta_{u}$, where $x^{i} \theta_{t} \eta_{u} \in H_{t u}^{i}=H_{i}^{i} \eta_{u}$, for all $i<\alpha$.

Lemma 3. If $x^{i} \in H_{t u}^{s}, x^{j} \in H_{s o}^{s}$, then $f(x)=e$, unless both $t=s$ and $u=v$.

If $f(\boldsymbol{x}) \neq e$, by (8), $u=v$, since $H_{t u}^{i} \subset H_{u}^{\prime i}$.

If $t \neq s, x^{i}=y^{i} \eta_{u}, y^{i} \in H_{i}^{i}$, and $x^{i}=y^{j} \eta_{u}, y^{j} \in H_{s}^{j}$. Defining $y^{k}$ to be $x^{k}$, for all $k \neq i$, $j$, we have, by (7), (8), (9), and Lemma 2,

$$
\begin{aligned}
& e=f(y)=\prod_{u \in U} f\left(y \eta_{u}\right), \\
& f\left(y \eta_{u}\right) \in K_{u}^{\prime} \quad(\text { all } u \in U) \text {, } \\
& f\left(y \eta_{u}\right)=f(x)=e .
\end{aligned}
$$

Lemma 4. If, for all $i<\alpha, x^{i}=\prod_{t \in T, u \in U} x_{t u}^{i}, x_{i u}^{i} \in H_{t u}^{i}$, then

$$
f(\mathbf{x})=\prod_{t \in T, u \in U} f\left(\mathbf{x}_{t u}\right) .
$$

This follows from Lemma 3 exactly as Lemma 2 from (8). The mutual commutativity of the $f\left(\mathbf{x}_{t u}\right)$ follows easily, by Lemma 1, from that of the $x_{i w}^{0}$.

The proof that, for $i \leqq \alpha, H^{i}$ is the direct product of the $H_{t u}^{i}$, that $H_{t u}^{t}=H_{t}^{i} \cap H_{u}^{\prime \prime}$, and that $f=\prod_{t \in T, u \in U} f_{t u}^{\prime \prime}$ now follows as for finite $\alpha$, and the uniqueness of the decomposition as a product of indecomposable mappings follows.

Related $\alpha$-linear mappings. We extend a known result on the homomorphisms of finite Abelian groups to $\alpha$-linear mappings ( $\alpha$ any ordinal of class 1 or 2).

Theorem 2. If $f$ and $g$ are $\alpha$-linear mappings on $\left(H^{i}, i<\alpha\right)$ to $K$, such that, for every $(\mathrm{x})$, there is a number $\lambda(\mathbf{x})$, such that

$$
g(\mathbf{x})=f(\mathbf{x})^{\lambda(\mathbf{x})},
$$

then, under suitable conditions, there is a number $\lambda$, such that, for all (x),

$$
g(\mathbf{x})=f(\mathbf{x})^{\lambda} .
$$

We may assume, without loss of generality, that (2) is satisfied by $f$, so that $H^{i}(i<\alpha)$ is Abelian and our main condition is that $H^{i}$ has a finite exponent $m$ (by (2), the same for each $i$ ). Then $H^{i}$ has a finite or infinite basis $\left(x_{j}^{i} ; j \in J^{i}\right)$. 
Denoting the order of $x$ by $[x], m_{j}^{i}=\left[x_{j}^{i}\right], m_{0}^{\imath}=m$, and we can take $\left[f\left(\mathbf{x}_{0}\right)\right]=m ; m(\boldsymbol{x})=[f(\boldsymbol{x})]$ divides $\left[x^{i}\right]$.

We now show that, if the theorem is true for all $\alpha$-linear mappings, it is also true for all $(\alpha+1)$-linear mappings.

Considering those $\mathrm{x}$ with $x^{\alpha}=a \in H^{\alpha}$, we have an $\alpha$-linear mapping on $\left(H^{i}, i<\alpha\right)$ to $K$ and, by hypothesis, we have $\lambda(a)$, such that

$$
g(\mathbf{x})=f(\mathbf{x})^{\lambda(a)}, \quad \text { whenever } x^{\alpha}=a .
$$

Since $g$ satisfies (1) and $f$ satisfies (1) and (2), it is easy to see that

$$
\left(x_{0}^{\alpha} x_{j}^{\alpha}\right)^{\lambda(a)}=\left(x_{0}^{\alpha}\right)^{\lambda_{0}}\left(x_{j}^{\alpha}\right)^{\lambda_{j}},
$$

where $a=x_{0}^{\alpha} x_{j}^{\alpha}$ and $\lambda_{j}=\lambda\left(x_{j}^{\alpha}\right)$. Then

$$
\lambda(a) \equiv \lambda_{0}(\bmod m), \quad \lambda(a) \equiv \lambda_{j}\left(\bmod m_{j}^{\alpha}\right),
$$

so that

$$
\lambda_{j} \equiv \lambda_{0}\left(\bmod m_{j}^{\alpha}\right) .
$$

It now follows easily that $\lambda=\lambda\left(\mathbf{x}_{0}\right) \equiv \lambda_{0}(\bmod m)$ satisfies (13). Since the result is known (or is proved similarly) when $\alpha=1$, it is true by induction for finite $\alpha$.

When $\alpha=\omega$, we use the limiting process and assume that $f$ and $g$ satisfy (5) and also that

$$
\lim _{n}\left(a_{n}^{\lambda}\right)=\left(\lim _{n}\left(a_{n}\right)\right)^{\lambda}, \quad a_{n} \in K .
$$

We define $\boldsymbol{x}_{n}$ by

$$
x_{n}^{i}=x^{i}, \text { for } n>i, \quad \text { and } \quad x_{n}^{i}=x_{0}^{i}, \text { for } n \leqq i .
$$

Thus $\lim \left(\mathbf{x}_{n}\right)=\boldsymbol{x}$, and then

$$
g(\mathbf{x})=\lim _{n}\left(g\left(\mathbf{x}_{n}\right)\right)=\lim _{n}\left(\left(f\left(\mathbf{x}_{n}\right)\right)^{\lambda}\right)=\left(\lim \left(f\left(\mathbf{x}_{n}\right)\right)\right)^{\lambda}=(f(\mathbf{x}))^{\lambda} .
$$

The general result now follows by transfinite induction.

\section{REFERENCE}

1. N. J. S. Hughes, The use of bilinear mappings in the classification of groups of class 2, Proceedings of the American Mathematical Society vol. 2 (1951) pp. 742-747.

University College, Leicester 